\title{
La dimensión del afecto y la influencia del psicoanálisis en la conceptualización del populismo de Ernesto Laclau
}

A dimensão do afeto e a influência da psicanálise na conceitualização do populismo de Ernesto Laclau

The affective dimension and the influence of psychoanalysis on Ernesto Laclau's conceptualization of populism

\section{Introducción}

Este artículo aborda la relación entre psicoanálisis y política, a partir del trabajo de Ernesto Laclau sobre el tema del populismo. La originalidad de este autor ha consistido siempre en darle a este concepto un estatuto teórico-político muy relevante, congruente con su valor social e histórico, y en ampliar el horizonte de investigación sobre el cual desplegar sus principales aristas y definiciones. Más allá de su connotación negativa - dado la elasticidad y vaguedad de su capacidad explicativa - ciertamente fenómenos políticos de fácil asociación con el populismo, como lo son el peronismo o el varguismo, han atendido y aún atienden a un interés genuino dentro de la ciencia política. No obstante, las insuficiencias de las herramientas teóricas que ella proporciona para su análisis son notables. Nuestra hipótesis respecto a dicha desavenencia entre teoría y práctica social se encuadra en la hipótesis de Ernesto Laclau

Doutoranda em ciência política pela Universidade de São Paulo. São Paulo, SP, Brasil. Bolsista FAPESP. 
cuando señala que "el populismo, como lugar de un escollo teórico, refleja algunas de las limitaciones inherentes al modo en que la teoría política ha elaborado la cuestión de cómo los agentes sociales 'totalizan' el conjunto de su experiencia política” (LACLAU, 2005, p.16). En las antípodas de aquellos que creen que habría que abandonar el concepto, Laclau sostiene que si es verdad que el populismo es el nombre de cierta imposibilidad de delimitación de una realidad social y política históricamente dada; si es válido afirmar que el populismo es una suerte de concepto comodín que la ciencia política tendría a mano para homogeneizar a todo un conjunto de eventos políticos que envuelven una alta movilización popular; en definitiva, si es el populismo un concepto con el que podemos explicar un abanico enorme de fenómenos políticos a lo largo de la historia, a la vez que esta misma elasticidad lo tornaría un concepto poco específico y ajustado, entonces esta es la razón por la cual, lejos de tener que rechazarlo, lo que la ciencia política debería hacer es ampliar el horizonte de su indagación.

Es en este simple razonamiento en donde, entendemos, reside en gran parte el mérito de Ernesto Laclau. Pues le permitirá abrirse a una interrogación sobre el populismo que es totalmente diferente de los abordajes clásicos. Y lo conducirá a considerarlo ya no como aquel concepto admitido porque excluido - de relevancia teórica, de capacidad explicativa, de rigurosidad científica etc.-, sino todo lo contrario: como un concepto que nos permite acceder al análisis del lugar sintomático de lo político en cuanto tal. El populismo sería un concepto sintomático porque pertenece a una problemática teórica más amplia que es el modo de constitución de las identidades sociales y políticas. Es decir, trabaja la cuestión de la formación de la subjetividad, la construcción del sentido de la realidad y el sentido de pertenencia de una determinada forma de comunidad. Laclau nos permite colocar la discusión teórica en un lugar menos incierto que aquel que proporcionan los análisis políticos al respecto cuya concepción de lo político se fundamenta tanto en términos de luchas o tensiones entre intereses de clase, como en la existencia de correlaciones de fuerza entre individuos 
más o menos orgánicamente organizados e incorporados a la representación institucional.

Revisar este concepto bajo el enfoque que proponemos nos conducirá a interrogar la identidad política en términos que envuelven una alteridad interna que frustraría la esperanza de poder apelar a la antinomia clase social/ individuo para definir al actor social arquetipo del accionar político propiamente dicho. Asimismo, Laclau nos proporcionará herramientas teóricas para comprender la razón por la cual el populismo ha recibido históricamente una pluralidad de sentidos, y hasta en algunos casos incongruentes entre sí. No porque podamos con su auxilio despejar la ecuación final. Muy por el contrario, porque su enfoque de la política se basa en la actuación de agentes sociales demandantes que constituyen su identidad política, y el espacio político propiamente dicho, sobre una falla estructural imposible de eliminar completamente y de modo definitivo en una sociedad. Lo que permite interpretar la pluralidad de sentidos que adquiere teóricamente el concepto como el reverso de situaciones sociales heterogéneas en las cuales de lo que se trata, sin embargo, es en todo caso de lo mismo: "taponar" contingentemente esa falta estructural a la identidad política con alguna conciencia, con alguna significación.

Un relevante punto en común entre Laclau y Lacan es la crítica - con sustantivas diferencias y distintos grados de elaboración conceptual, claro está - que en ambos encontramos a la noción de individuo, si le adjudicamos la racionalidad que subyace del pensamiento político moderno. Concebir la identidad como falta, incompletitud, desarmonía, incongruencia se opone a la idea de aquella racionalidad que escinde al individuo en el punto exacto donde comienza a actuar su sensibilidad, "animalidad" o no-saber. Esta clase de escisión entre razón y sentidos, racionalidad e irracionalidad, materialidad y espiritualidad la podemos encontrar en el pensamiento político contractual, en la filosofía kantiana, en la dinámica de las clases sociales de Marx, en la teoría de la acción social de Max Weber, y, en general, en las corrientes del pensamiento social y filosófico que, de alguna manera, recuperan 
algo de ese sujeto levantado por el Iluminismo. Es precisamente esta dualidad que el psicoanálisis de Freud subvierte, revolucionando el pasaje del siglo XIX al XX, a partir del descubrimiento del inconsciente. Asimismo, es a través de la incorporación de la teoría psicoanalítica que el marxismo "rejuvenece" bajo la crítica cultural e ideológica de los modos de dominación capitalista, recuperando algo de ese sujeto descentrado que desentona con la racionalidad inaugurada con el Iluminismo.

En La razón populista, Ernesto Laclau intentará comprender la identidad política popular como el resultado de una articulación tal que imposibilita aprehenderla en términos de conciencia individual o de clase homogénea. El actor político del populismo es el pueblo. Y el pueblo surge de la articulación de una voluntad política constituida bajo lo que Laclau denomina "lógica hegemónica". Y en el despliegue de esta lógica hegemónica el líder populista tiene un papel fundamental. Por eso es que este artículo pretende detenerse específicamente en el análisis de esta función. En la primera parte ubicamos el enfoque posmarxista de Laclau dentro de la teoría política contemporánea como formando parte de la corriente posestructuralista. ¿Cuál es la deconstrucción específica sobre la teoría marxista operada por Laclau? Veremos que la misma apunta a combatir el esencialismo de la definición de las clases sociales, reformular el modo como el marxismo piensa la constitución de la identidad y la actuación política. En la segunda parte damos cuenta, resumidamente, de su elaboración conceptual del fenómeno del populismo para detenernos específicamente en el papel que juega el líder populista en ese proceso de construcción hegemónica de la identidad política popular. Finalmente, establecemos un análisis crítico respecto de la analogía que Laclau establece, para describir y ponderar la importancia de la función del liderazgo en la hegemonía populista, entre el líder populista y el objeto $a$. ¿Qué puede significar decir que el líder populista tiene para la teoría de la hegemonía un valor teórico equivalente al del objeto a para la teoría psicoanalítica de raigambre lacaniano? Esta es la pregunta que el artículo pretende explorar con mayor detenimiento. 


\section{La crítica posmarxista de la perspectiva teórico-política de Ernesto Laclau}

Hay un cierto recorrido de indagación y formulación teórica de Laclau que, entendemos, es el que mejor nos proporciona la clave de lectura para comprender lo que él entiende por hegemonía populista y el lugar que en la misma le asigna al liderazgo político. Ese recorrido, que va del libro Política e ideología en la teoría marxista: capitalismo, fascismo y populismo (1979) a la publicación de La razón populista (2005), no es más que un recorrido diacrónico. Sin embargo, este primer trabajo de Laclau suele pasarse por alto; en parte, por ser un texto que todavía pertenece a un campo de indagación marxista, que luego Laclau abandonará; en parte, por la relevancia que tuvo la publicación escrita en coautoría con Chantal Mouffe, Hegemonía y estrategia socialista: hacia una radicalización de la democracia (2004), el cual se tornará muy rápidamente un clásico de la ciencia política contemporánea. Para nosotros, en cambio, ese primer trabajo de Laclau representa un punto de partida imprescindible porque encontramos aquí ya el establecimiento de los interrogantes y las críticas fundamentales a la teoría marxista que van a acompañarlo a lo largo de toda su producción intelectual.

Por su parte, el segundo trabajo que mencionamos es sin lugar a dudas un punto de llegada, que tiene como referencia aquel primer libro como su punto de partida. La razón populista es el momento más acabado que la elaboración teórica de Laclau consiguió desarrollar para pensar el tema del populismo. Y claro que decir esto no significa simplificar las cosas a tal punto de entender que todo lo que el autor pensó y publicó en el medio, o inclusive después de escribir este libro, es una mera mediación o un simple anexo que auxilia a la reflexión sobre el populismo. Pero es conveniente comenzar por el trabajo de 1979 porque ahí se sientan las bases para una crítica a la teoría marxista y al modo en que ella piensa a la acción política. En ese sentido, debemos entender que el posestructuralismo que encontramos en Laclau es el posmarxista. Y si su trabajo contribuyó para deconstruir de alguna manera al estructuralismo, ello se debe a que su crítica al marxismo apunta a ser una crítica a una teoría 
moderna entendida, leída como estructuralista. Con relación a ello, ¿cuáles son las principales preguntas críticas que Laclau le formula al marxismo?

El principal cuestionamiento de Laclau es que el marxismo no puede pensar la categoría de "pueblo"; y por lo tanto, no puede pensar la subjetividad política más allá de la definición de las clases sociales prefiguradas por la actividad económica, así como tampoco la emancipación de la opresión social más allá de la cristalización de la lucha de clases. El estructuralismo marxista al que apunta la crítica de Laclau se caracteriza por el hecho de ser una teoría social que entiende que la sociedad capitalista es una totalidad dialéctica, compuesta por una estructura (la economía, es decir, el ámbito de la producción social de la riqueza material) que determina a la superestructura (ideología, religión, derecho, filosofía, etc. pero también, y sobre todo, la política y el Estado). Considera que la dominación material de una clase sobre el resto de la estructura social explica la dominación espiritual ejercida en el plano de la superestructura, haciendo pasar sus intereses particulares de clase como el interés general de la sociedad. Recordemos que lo que para Marx caracterizara a la estructura material en la sociedad capitalista es una contradicción, "la contradicción fundamental", entre las relaciones sociales de producción (la existencia de capitalistas propietarios de los medios sociales de producción vs los trabajadores "libres" que venden su fuerza de trabajo) y el desarrollo histórico de las fuerzas productivas. Con relación a la relación entre estructura y superestructura, y al "enmascaramiento" que caracteriza a la segunda -por presentar el interés de la clase dominante como el interés común-, una de las consecuencias principales para pensar la política es entender que para el marxismo no hay manera de transformar la sociedad sin transformar radicalmente la estructura. Es decir, sin resolver la contradicción fundamental que explica y orienta, en última instancia, la manera injusta de producir y distribuir socialmente la riqueza.

La teoría de la revolución de Marx depende directamente de su teoría de la historia: el materialismo histórico. La idea básica 
de la concepción marxista de la historia es que cada corte establecido con el modo de producción social predominante a lo largo de la historia (mercantilismo, feudalismo, capitalismo, y así deberá también suceder con la implantación del comunismo a nivel global) se ha caracterizado por haber resuelto la contradicción principal en la dirección de un cambio absoluto de las relaciones sociales de producción, en particular, y en la forma de organizar la sociedad, en general. Del mismo modo en que para Marx la división social entre opresores y oprimidos a lo largo de la historia de la civilización estuvo siempre determinada por esta contradicción entre el desarrollo de las fuerzas productivas y las relaciones sociales que las frenan, el motor de la historia es la lucha de clases que tiende a superar esta contradicción principal. En ese sentido, junto con la determinación de los agentes sociales a partir de su participación específica dentro del sistema de producción, lo que Marx conjuntamente define es una suerte de "misión histórica" de las clases sociales destinadas a superar dicha contradicción. La burguesía, por el papel histórico que tuvo en el desarrollo de las fuerzas productivas, fue la clase social en la que se encarnó la contradicción entre el desarrollo económico y las relaciones sociales feudales de producción que lo frenaban, y por eso su destino fue revolucionar a la sociedad feudal. Del mismo modo, y por la misma razón, el proletariado sería la clase social destinada a superar el modo de producción capitalista y a la sociedad burguesa. En el marco de este esquema conceptual marxista, que nosotros estamos simplificando enormemente, retomemos la pregunta por el populismo y la crítica de Laclau.

El problema principal con el populismo, en clave con el análisis de Marx del bonapartismo, es que impide la cristalización de la contradicción principal que caracteriza a la sociedad capitalista $y$, de este modo, obstaculiza la lucha de clases como forma de eliminación de la propiedad privada de los medios sociales de producción y la superación de la dominación de clase. En este sentido, lo que Laclau defiende es que la condición de la clase proletaria no alcanza para imponerle un papel histórico revolucionario, si antes no se reconoce que lo que opera con fuerza en la dominación de 
clase no es tanto la determinación económica de la misma como la no identificación subjetiva del proletariado como clase oprimida. Para Laclau la existencia de la explotación no es garantía suficiente para la adjudicación de un papel histórico-político revolucionario (reactivar la lucha de clases), dado que la acción política subversiva no comienza con la condición efectiva de ser explotado sino con la resistencia a dicha explotación. El punto central aquí es entender que para Laclau esta resistencia se puede construir de maneras distintas, porque depende no tanto de la contradicción que expresa la explotación capitalista como de actos de interpelación discursiva e identificación subjetiva que no necesariamente coinciden con la lucha de clases entre el proletariado y la burguesía. Y es alrededor de esta disconformidad teórica con el marxismo que se articulará todo el esfuerzo de Laclau y Mouffe de los años 80 por reformular el concepto de hegemonía de Antonio Gramsci, de modo tal de poder volver a pensar a la izquierda y las alternativas políticas emancipatorias posibles frente a la dominación del capitalismo, luego de la caída de la Unión Soviética. Es éste, igualmente, el mismo impulso intelectual de Ernesto Laclau a la hora de repensar el concepto de populismo.

\section{La hegemonía populista y la dimensión del afecto en la construcción del liderazgo político}

¿Quién es el pueblo para Laclau? ¿En qué consiste el populismo? Para este autor el populismo es una forma de constitución de la identidad política, la cual debemos entender como siendo básicamente negativa, vacía, sin fundamento ontológico. Y el pueblo es la figura que la encarna, es decir, la figura que permite la articulación y cristalización de la misma. Entender de este modo a la identidad política - como una construcción - obliga a Laclau a depararse con el enorme trabajo intelectual de tener que dar cuenta de las consecuencias analíticas que conlleva para pensar la política el hecho de asumir la importancia decisiva del discurso para la conformación de cualquier entidad inteligible. Ese es el motivo principal de su diálogo con el posestructuralismo, con Heidegger, Wittgenstein y 
también lo que en parte explica su interés por el psicoanálisis de Lacan y su relectura de la obra de Freud.

En ese sentido, teniendo en cuenta que el "pueblo" es un actor político construido discursivamente, debemos comenzar señalando que su expresividad mínima o el germen de su devenir es lo que Laclau denominó la demanda social. Para ser más precisos, la materia prima de la construcción simbólica de la figura del pueblo que opera políticamente en el populismo es la demanda social popular insatisfecha. Es decir que para Laclau no existe populismo, ni momento populista de la política sin que puedan visibilizarse y diferenciarse en la sociedad demandas sociales concretas que tiendan a desequilibrar el orden social imperante. El supuesto invariable del que parte, el condicionamiento para la emergencia del populismo, es que existan en la sociedad diversos tipos de demandas sociales que perturben, de alguna manera, la continuidad armoniosa de la sociedad. El poder político al cual van direccionadas estas demandas sociales, o bien las atenderá, o bien seguirá ignorándolas. A medida que la movilización socia avanza, esas distintas demandas sociales no atendidas - como podrían ser el reclamo por mejoras en el sistema educativo, la salud pública, la seguridad social, etc.- empiezan a tornarse equivalentes entre sí.

La articulación entre las distintas demandas sociales, que se asemejan porque no encuentran canalización en el actual sistema de representación institucional, es lo que define paralelamente una frontera social interna de la cual resulta una sociedad políticamente dividida en dos campos opuestos: un adentro y un afuera de la representación actualmente legítima. La distancia irreductible que separa la experiencia social de la disconformidad y el régimen de la representación legítima ya institucionalizado -demandas populares insatisfechas vs. el poder hostil a ellas-comienza a definir un antagonismo político en el cual "los responsables de que la plenitud de la comunidad sea precisamente el reverso imaginario de una situación vivida como ser deficiente, no pueden ser una parte legítima de la comunidad; la brecha con ellos es insalvable" (Laclau, 2005, p.113). La definición del antagonismo político que polariza el campo social 
presupone el privilegio de algunos significantes que condensan en torno de sí la identidad popular. Estos significantes nombran al conjunto en tanto cadena equivalencial significativa totalizante, al mismo tiempo en que excluyen de la propia identidad al adversario con el cual se antagoniza (es decir, nombran una alteridad interna).

Si bien las demandas sociales insatisfechas corporizan lo que excede a la representación posible dentro del sistema institucional, no consiguen unificarse espontáneamente puesto que sus especificidades pueden provenir de naturalezas muy diversas. La operación de dislocación por la cual un significante particular, esto es, un elemento diferencial de la serie de demandas sociales heterogéneas, pasa a asumir la representación de la totalidad de la cadena, homogeneizándola, es lo que Laclau define como hegemonía. En otras palabras, para que lo social se unifique es necesario de un desnivel, que se expresa cuando una particularidad asume la representación de esa totalidad de demandas sociales insatisfechas imposible de representar directamente. Es decir, "cuando una plebe (particularidad) reclama ser el único pueblo (universalidad abstracta) legítimo (hegemónico)" (LACLAU, 2005, p.108; los paréntesis son agregados nuestros). De esta manera, si las demandas sociales alcanzan un cierto grado de articulación y movilización política - dado que al comienzo no pasaran más allá de un sentimiento vago de solidaridad entre sí - pueden llegar a unificarse en una subjetividad más amplia y cristalizarse en un sistema estable de significación. En este momento estaríamos asistiendo a la constitución de una identidad política popular que es algo cualitativamente distinto a la suma de los lazos equivalenciales forjados en la coyuntura del primer momento. Los significantes que privilegiadamente consiguen nombrar al conjunto, representar al movimiento social reivindicatorio como una totalidad hegemónica, Laclau los denomina significantes hegemónicos o vacíos.

Por otro lado, Laclau afirma que esta operación de dislocación, necesaria para la totalización de la representación de la identidad política (es decir, para la cristalización del pueblo), sólo es posible gracias al componente "afectivo", a la "investidura radical" de ese 
nombrar bajo una demanda global el conjunto o la cadena de demandas sociales insatisfechas. Pero las demandas sociales que están en la base de toda configuración hegemónica del tipo populista no consiguen unificarse automáticamente, como hemos dicho anteriormente, dado que pueden promover reclamos de naturalezas muy diversas. Por lo cual es importante en este momento detenerse a analizar el elemento determinante para la homogeneización de la identidad popular: la productividad social del nombre de la hegemonía. Según Laclau, de este elemento depende la unidad de la identidad popular. Si la solidaridad inicialmente débil entre las distintas demandas democráticas puede darse por el hecho de compartir la condición de la insatisfacción, por el hecho de encarnar el fracaso de la representación del sistema institucional vigente; en un segundo momento esa ligazón original deberá dar paso a una articulación singularizante que depende enteramente de la producción social de un nombre que la (re)funde.

En un apartado sumamente complejo de La razón populista, titulado "Nominación y afecto", Laclau nos provisiona de ciertas pistas que nos permitirán comprender mejor esta su perspectiva fundacional y performativa de la operación de nominación. Inspirándose en la polémica abierta dentro de la filosofía analítica contemporánea entre teóricos descriptivistas y anti-descriptivistas, acerca del modo en el que los nombres se relacionan con las cosas, Laclau profundiza el sentido del abordaje discursivo de la política, abandonando, de cierta manera, la postura contextualista adoptada en sus primeros trabajos, para dar lugar a una perspectiva anti-descriptivista más radical. Para este autor el elemento que brinda la materialidad real a la subjetividad política es el nombre en tanto significante cada vez más autonomizado. Esta radicalización del estructuralismo lingüístico le permite a Laclau postular la tesis que afirma que el nombre acaba tornándose, retroactivamente, el fundamento de la cosa. En términos políticos diríamos que el nombre que consigue articular la cadena de demandas iniciales termina de configurar una identidad política popular al momento que puede identificar, en retrospectiva, aquéllas demandas como 
propias, como las demandas sociales que alza como "bandera" este determinado colectivo político. A esta operación de nominación, momento cualitativamente diferente y fundacional, Laclau la llama también "investidura radical" en un objeto parcial de una totalidad mítica inconmensurable consigo misma, o "el hacer de un objeto la encarnación de una plenitud mítica” (LACLAU, 2005, p.148).

Más adelante en el texto vamos a reconstruir los posibles retazos del psicoanálisis allí presentes, para analizar hasta qué punto esta asociación es pertinente o no. Por el momento es importante apenas retener esta asociación entre "nominación" e "investimento", y citar al autor para terminar de despejar el sentido que guarda la nominación en el proceso de configuración de las identidades políticas:

La unidad del conjunto equivalencial, de la voluntad colectiva irreductiblemente nueva en la cual cristalizan las equivalencias particulares, depende enteramente de la productividad social del nombre. Esa productividad deriva, exclusivamente, de la operación del nombre como significante puro, es decir, no expresando ninguna unidad conceptual que la preceda (como sería el caso si hubiéramos adoptado una perspectiva descriptivista). (LACLAU, 2005, p.139, el destacado es del autor).

Al mismo tiempo, el autor afirma que en este proceso de nominación la fuerza del componente afectivo con la que un significante nombra al pueblo es la dimensión fundamental de su investidura radical: lo que posibilita a un objeto parcial, a un elemento diferencial asumir la representación de una plenitud que, como tal, siempre será mítica. En otras palabras, el componente afectivo vehiculizado por el nombre que encarna, parcial y coyunturalmente, al pueblo es el elemento que sobredetermina a la identidad política en cuanto tal. Pero acá es donde debemos destacar que la idea de sobredeterminación de Laclau, al igual que en Althusser, no puede disimular el hecho de que, de cierta manera, es heredera del sentido que Freud le otorga dentro de la práctica psicoanalítica. Hay muchas intuiciones teóricas de Laclau, en particular sobre aquellos problemas que él 
identifica en la teoría marxista y en la concepción hegeliana de la historia, que comienzan a despejarse a partir de esta idea de sobredeterminación. Sin embargo, consideramos que la exploración de esta categoría no fue lo suficientemente exhaustiva, en el sentido de no haber logrado abrir camino a las respuestas requeridas. De hecho, muchos de los obstáculos que su teoría política tiene para establecer un diálogo con el psicoanálisis, por ejemplo cuando relaciona la nominación y el afecto con los conceptos lacanianos de "goce", punto de almohadillado o el objeto a, creemos, se originan en el arrastre de esta deuda. Para nosotros, la noción de sobredeterminación es inseparable de la nominación y del afecto, justamente porque se refieren a eso que él llama una "productividad social". $\mathrm{O}$, dicho de otro modo, la sobredeterminación que se le imputa a las identidades políticas no es un dato anterior sino más bien el resultado o efecto de una ligación particular entre lo que Laclau denomina la nominación y el afecto, que se ubica al nivel de la base social del populismo.

La noción de sobredeterminación, al estar así estrechamente vinculada a la nominación y el afecto, guarda alguna relación con la perspectiva económica freudiana de las pulsiones, que supone la posibilidad de transferir o estimar/desestimar el valor de representación (catexia) de algunos elementos significantes, al mismo tiempo que produce un "estado de eclipse" que le permite al significante independizarse o sustraerse del significado. El afecto, entonces, "eclipsa" al lenguaje y el nombre del líder "eclipsa" a la identidad política popular; al mismo tiempo, la productividad social que está en su base imprime en el nombre propio una significación adicional, la cual ubica al movimiento político en un más allá de la dimensión "personal" del líder. Resumiendo lo anterior, podemos decir entonces que el afecto es, en primer lugar, lo que amalgama a la insatisfacción de las distintas demandas sociales y las torna articulables. En segundo lugar, el afecto no es independiente del lenguaje sino que se soporta únicamente en él y viceversa. 
El afecto no es algo que exista de por sí sólo, independientemente del lenguaje, sino que sólo se constituye a través de la catexia diferencial de una cadena de significación. Esto es exactamente lo que significa 'investidura' [...] Cualquier totalidad social es el resultado de una articulación indisociable entre la dimensión de significación y la dimensión afectiva (LACLAU, 2005, p. 143).

Más adelante Laclau establece una analogía entre esta traducción de la dimensión afectiva que el discurso produce en términos de una "investidura radical" y la función de encarnación parcial en el objeto $a$ lacaniano de una plenitud mítica perdida.

No existe ninguna plenitud social alcanzable excepto a través de la hegemonía; y la hegemonía no es otra cosa que la investidura, en un objeto parcial, de una plenitud que siempre nos va a evadir porque es puramente mítica (en nuestras palabras: es simplemente el reverso positivo de una situación experimentada como "ser deficiente"). La lógica del objeto $a$ y la lógica hegemónica no son sólo similares: son simplemente idénticas. (LACLAU, 2005, p. 148)

En el caso del populismo, el que asume la función de ser un objeto que permite la homogeneización, parcial y contingente, de la representación de la experiencia política es el líder populista. Pero el punto importante que queremos destacar es que debemos separar el líder del liderazgo, y entender que el líder es el nombre de quien viene a ocupar o encarnar el lugar del liderazgo. Un lugar de poder que, de cierta manera, es construido con anterioridad a su emergencia pues depende de la base social que se encuentra determinada por la situación de insatisfacción de sus propias demandas. Es decir que el liderazgo político es un lugar vacío, el lugar enunciativo desde donde se legitima el discurso que representa a la experiencia política, que de cierta forma es independiente del nombre que coyunturalmente lo pueda encarnar. De esta manera podemos entender mejor la analogía con el objeto $a$ de Lacan, en lo que se 
refiere a la encarnación de la función de representación parcial y contingente del objeto de deseo en el lugar de la renuncia al acceso a una plenitud de goce absoluto. ¿Por qué? Al separar el nombre del líder de la función que cumple el lugar del liderazgo podemos entender mejor la analogía del objeto $a$ de Lacan por el siguiente motivo: porque el objeto $a$ es el objeto que viene a regular el deseo del sujeto una vez que éste ingresa en el universo simbólico, en el campo del Otro, a partir de la renuncia a un objeto de goce pleno (la Cosa freudiana, que para el niño pequeño suele ser la madre).

La analogía de Laclau está relacionada con que para él el nombre del líder populista, por ser el que permite la representación hegemónica de la identidad política popular, que es siempre parcial y contingente, de entrada está cumpliendo una función que se articula sobre la base de una renuncia: la renuncia a la representación plena o mítica de lo social no atravesado por el antagonismo político. En este sentido, lo primero que hay que entender es que la analogía establecida por Laclau es entre el lugar del liderazgo político y el lugar de la enunciación del Sujeto barrado (o atravesado por la represión primaria) que adviene en el campo del Otro. En segundo lugar, lo que la analogía de Laclau pone en equivalencia es la Cosa freudiana, la fuente de goce absoluto que la madre representa para el cachorro humano, y la plenitud mítica de lo social, entendida como la reconciliación política plena y definitiva (lo que en el lenguaje común podemos identificar con expresiones como "la unión de todos los brasileros" y cosas por el estilo). En tercer lugar -y este es para nosotros el punto más polémico, pero que es inmanente a la analogía- el nombre del líder que encarna el lugar y la función del liderazgo político es equiparado con el estatuto parcial del objeto de la pulsión. Y aquí es donde la cosa se complejiza y debemos, necesariamente, establecer un diálogo más demorado con el psicoanálisis como para que la analogía de Laclau no se pierda en un mar conturbado de significaciones incongruentes.

Para Laclau todo acto de lenguaje o proceso de nominación comporta siempre una sobredeterminación, una investidura, un afecto, un plus-de-gozar que, por otra parte, no siempre queda 
muy bien articulada en la teoría, y sin embargo es capital para comprehender la especificidad de la perspectiva de lo político que permea su concepto de populismo. Tomar el "afecto" como la dimensión que explica la performatividad presente en todo proceso de nominación, o como el elemento que sobredetermina el populismo - es decir, esta lógica específica de devenir los agentes sociales en sujetos políticos -, es un aspecto en la teoría de Laclau muy importante, aunque no siempre queda claro el sentido en el que trabaja. Por ello es necesaria una lectura crítica de esta analogía elaborada por Laclau.

\section{Entre la demanda insatisfecha y la falta constitutiva de la identidad política: una aproximación crítica a la asimilación del concepto de objeto $a$ en la conceptualización del populismo}

En esta parte del texto vamos a aproximarnos de la conceptualización del concepto de objeto en la teoría psicoanalítica, tomando como referencia principal el trabajo de Diana Rabinovich. Es importante, no obstante, aclarar que este breve desarrollo no pretende ser exhaustivo, sino un punto de apoyo sobre el cual más adelante podamos pararnos para volver sobre la teoría política de Laclau e intentar reinterpretar su analogía conceptual entre el objeto a lacaniano y el lugar y la función que ocupa el líder en la hegemonía populista.

La primera cosa que debe quedar en claro de entrada para abordar la noción de objeto es que para el psicoanálisis el ser humano, antes de ser sujeto, nace como objeto. Y que la subjetividad se constituirá en un segundo tiempo. El niño pequeño es o no es, primeramente, un objeto de deseo: del deseo de los padres, de los progenitores o de algún miembro de la familia o seno social en el cual este nace. Todo ser hablante viene al lugar de un deseo o no-deseo de hijo. Pero al ser un acontecimiento que es producido en el marco de un contexto social, cultural, político, afectivo familiar, en definitiva, simbólico particular, por ese motivo al mismo tiempo el niño en el lugar de objeto de deseo es destinado a la entrada en un proceso de 
subjetivación que se inicia, inclusive, antes de su nacimiento. ${ }^{2}$ Pues, para que advenga el sujeto en ese ser hablante, inicialmente mero objeto del deseo de hijo, es necesario el campo del Otro, es decir, el lenguaje y el lazo social que se sostiene en el discurso.

Determinado por el desamparo y la impotencia que caracterizan al cachorro humano, el niño pequeño, al mismo tiempo, toma a la madre como primer objeto en tres sentidos diferentes. En primer término, la madre es para el bebé el objeto de deseo freudiano, el objeto perdido, en la medida que es el Otro primordial que por estructura permite el surgimiento del y el acceso a la realidad simbólica misma. En segundo término, la madre también coincide en determinado momento con lo que Freud distingue como objeto de la pulsión parcial. En tercer lugar, la relación con la madre definirá, en la triangulación edípica, la elección del objeto de amor del sujeto, que lo identificará, posteriormente, en una u otra dirección de la identidad sexual (homo- hetero). Todo esto que desarrollaremos no deja de recordarnos ese famoso dicho popular que dice "madre hay una sola”, a lo que contestamos: ¡Por suerte!

Por un lado, es ese Otro inolvidable que en función del desamparo y la indefensión permite el surgimiento del objeto de deseo como diferente al objeto de la necesidad. Por otro, se articula simultáneamente con la pulsión parcial -hecho particularmente claro en relación al pecho como objeto pulsional-, y con el complejo de Edipo, en el que juega el papel central en tanto "persona" amada, es decir, como objeto total. (RABINOVICH, 2007, p.22.)

Decíamos que las diferencias conceptuales que existen con relación al concepto de objeto en la teoría psicoanalítica eran

2 A través de los rituales y las acciones orientadas a darle un lugar a ese deseo en la propia vida del ser deseante. Ejemplos típicos de este proceso son la organización de un lugar especial para el niño en la propia casa, la adquisición de objetos que irán a satisfacer sus necesidades teniendo en cuenta su propio rasgo -el género femenino o masculino, por ejemplo-, las imágenes personales y sociales fantaseadas alrededor del futuro bebé, las características de su personalidad, etc., y, claro, algo muy importante, la elección de su nombre propio. 
apuntadas por Rabinovich por el hecho de que la madre, como Otro primordial es para el niño pequeño objeto en tres sentidos diferentes. Entonces, repasemos esa diferencia, para ver de qué modo podemos entender el concepto de objeto para pensar la política en el uso que le da Laclau. El punto de partida para entender el concepto de objeto para la teoría psicoanalítica, según Rabinovich (2007), debe ser entonces la formulación del objeto perdido freudiano. ¿Qué es el objeto perdido? Como lo señaláramos recién, anterior al surgimiento del sujeto, a que podamos decir de él que puede apropiarse de cierto lugar de la enunciación, hay una condición de pérdida estructural en el ser hablante que es lo que le permitirá el acceso a la representación de la realidad misma: la pérdida del Otro primordial, la llamada Cosa, según Freud. Esa pérdida es la que abre el camino a una búsqueda infinita caracterizada por estar comandada por el deseo de re-presentar, en el sentido de re-encontrar algo que por estructura y desde siempre está perdido. Esa condición de posibilidad estructural, de la pérdida real del Otro primordial para el establecimiento de la representación de la realidad - de cuya presencia el infante humano inicialmente depende para poder ver satisfechas sus necesidades y pulsiones vitales - deja una marca o huella imborrable que orientará la búsqueda incansable del sujeto hacia el reencuentro con ese Otro inolvidable, paradójicamente, perdido aunque nunca poseído.

Sobre el telón de fondo de una nostalgia, de un anhelo de la búsqueda del encuentro primero con ese Otro, encuentro para siempre perdido, se instala una huella mnésica, esa re-presentación, que nunca alcanza la presencia anhelada. La huella es pues solidaria de una pérdida y constituye una memoria orientada en sus recorridos, en su búsqueda, por el principio del placer y su meta a nivel primario, la identidad de percepción. Memoria que busca la repetición de una 
percepción imposible, que la alucinación simula pero no alcanza. (RABINOVICH, 2007, p.14.) ${ }^{3}$

Esa pérdida estructural en los seres hablantes instala lo que en psicoanálisis se denomina una hiancia, una distancia infranqueable entre la experiencia de satisfacción que el objeto mundano produce y la alucinación de la percepción creada al momento de la satisfacción primera. Satisfacción causada, fundamentalmente, no por la cancelación de la necesidad (en un sentido meramente orgánico) sino por la respuesta que la presencia del Otro hace posible. Esa hiancia, falta, agujero estructural entre la experiencia de satisfacción que los objetos mundanos canalizan y la percepción que de eso el infante tiene por primera vez, y que guardará en la memoria, marca y distingue para siempre a los seres hablantes de otras sociedades animales propiciando un nuevo estatuto para todo lo que sea del orden de las necesidades "biológicas" humanas, justamente porque la propia percepción de la experiencia de satisfacción precisa de palabras o de una traducción a nivel significante, es decir, supone ya el campo del Otro.

El desamparo humano, al determinar la impotencia del infans, da a ese otro su lugar y su función primordial, creando así una nueva "necesidad" -término que debe entenderse en su doble sentido en castellano, biológico y lógico-, necesidad lógica entonces que es tan exigente y tan imperiosa como la necesidad biológica, necesidad lógica de la dimensión de ficción propia del deseo en tanto que humano. Ficción y realidad psíquica no se oponen, hambre de signos podría llamárselas, de signos de la presencia de ese otro inolvidable, rastro engañoso de una presencia imposible de conjurar. (RABINOVICH, 2007, p.14.)

3 Asimismo, si esta falta de naturalidad entre el ser humano y el objeto de su satisfacción deja una huella mnésica que orientará el camino hacia la realización metonímica del deseo, regido por el principio del placer, el dolor también deja tras de sí una marca indeleble que Freud llamó huella del objeto mnémico hostil (RABINOVICH, 2007, p.15). 
En otra clase teórica de la psicoanalista Rabinovich encontramos un ejemplo ilustrativo de esa nueva necesidad para el ser hablante, el "hambre de signos", cuando nos habla de aquellos objetos que permiten establecer soluciones "culturales" o artificiales para las necesidades "biológicas".

El pecho materno, para tomar la experiencia de satisfacción freudiana, se vuelve objeto mucho más allá de que calme o no el hambre. Lo que Freud definió como su primera teoría de la pulsión, la teoría anaclítica de la pulsión, del apoyo de la pulsión sobre la necesidad, hace que de allí en más el objeto humano siempre pueda ser sustituido por otro objeto. Plantear esto es lo mismo que decir que nada tiene significado fijo para el ser que habla, porque el chupete reemplaza a la madre, al seno materno, si la voz de la madre, ya no el chupete, reemplaza o puede sustituir y calmar al bebé que llora. Entramos en una dimensión en la cual el objeto es evidentemente simbólico y en tanto simbólico se caracterizará por ser siempre otro, por la posibilidad misma de ser sustituido por otro objeto. (RABINOVICH, 1986.) ${ }^{4}$

Rabinovich sostiene que lo que para Freud eran los llamados objetos parciales (pecho, heces, falo, etc.), esos que regulan la economía pulsional del sujeto, para Lacan no son más que objetos simbólicos -y por eso él podrá agregar posteriormente a la lista freudiana de objetos parciales, la voz y la mirada. ¿Qué significa esto? Que en la medida en que la madre habla, instala en el lugar de la pura necesidad biológica del niño pequeño el lugar del código. Vemos, entonces, que en el concepto de objeto que se desprende de la formulación de Freud del objeto perdido el significante hace su irrupción de manera radical, dejando en todos los seres hablantes esa especie de "hambre de signos". Es decir, instala la necesidad (lógica y biológica) de tener que enfilar junto con otros significantes

4 El destacado en esta y las siguientes citas de Rabinovich nos pertenece. 
para emprender esa búsqueda del placer que pueda alucinar el anhelado reencuentro primero (perdido, por estructura). En este caso, la condición de la pérdida del Otro necesaria hace referencia a la pérdida de ese goce absoluto que el niño pequeño experimenta cuando apenas es un puñado de pulsiones que se satisfacen con su sola presencia. ¿Cómo se da esa pérdida? El ir y venir de la madre, por ejemplo cuando atiende sus compromisos laborales o deseos personales, es lo que permite el establecimiento de una falta o agujero en el campo pulsional del goce absoluto del niño pequeño. Es esta falta de goce la que permite decir que el significante cava un agujero en la dimensión psíquica de lo real. De este modo, por culpa del significante, la satisfacción de la necesidad se torna para siempre una búsqueda constante por encontrar unos objetos simbólicos que vehiculen satisfacción, no desde el punto de vista del valor que los objetos pueden tener en sí mismos, sino por la experiencia alucinatoria de estar frente a ese Otro inolvidable que nunca nadie igualará. Cuando esa ficcionalización del re-encuentro a través del objeto es lograda; cuando se alcanza a identificar en un determinado objeto cierto re-conocimiento del Otro primordial, desde ese momento la satisfacción de la necesidad debe traducirse por el placer que otorga la realización del deseo. Y el objeto pasa de ser el medio de una necesidad supuestamente biológica o natural a la vía de la realización del deseo. Medio y vía no son lo mismo. Y aquí dimos con algo que nos permitirá introducir la definición del objeto parcial de la pulsión freudiano.

Según Rabinovich, al concepto freudiano de objeto parcial sí le corresponde la satisfacción como meta. Es decir, el objeto parcial de la pulsión tiene la función de satisfacer, neutralizar o canalizar el saldo de libido en contra del sujeto, ese que le impide la homeostasis o el equilibrio orgánico en sentido económico. En otras palabras, el fin de la pulsión de muerte es el retorno incesante al mismo lugar: el de la satisfacción. Ese volver "siempre al mismo lugar" de estos objetos de la pulsión está vinculado con la circularidad propia de la satisfacción que tiene como meta. En oposición a la realización del 
deseo, con la forma desplazada o metonímica que dibuja el objeto de deseo.

El objeto es aquí instrumento de la satisfacción, aquello con lo cual se obtiene la satisfacción y en tanto instrumento es precisamente el aspecto más variable de la pulsión: “(...) no está enlazado originariamente con ella, sino que se coordina con ella sólo a consecuencia de su aptitud para posibilitar la satisfacción. No necesariamente es un objeto ajeno; también puede ser una parte del cuerpo propio. Este papel instrumental lo hace apto por ende para satisfacer varias pulsiones. (RABINOVICH, 2007, p. 24.)

Vemos aquí que, para Freud, si hay algo variable en los objetos de la pulsión es el hecho de que un mismo objeto pueda satisfacer o tornarse medio de resolución de diversos problemas económicos pulsionales. Esa versatilidad característica del objeto de la pulsión parcial tiene su contrapunto en el concepto de fijación. Es decir, su lado patológico, de cierta manera, se establece cuando el círculo entre pulsión y objeto se torna extremadamente íntimo. Y más allá de que el ser hablante sea capaz de conjugar deseo y goce -es decir, hacer convivir dos funciones de objetalidad diferentes en un mismo objeto -, existe una diferencia importante entre el vínculo con un objeto que se torna versátil a la pulsión y la fijación de la pulsión en un objeto imprescindible al que el sujeto no puede de ninguna manera renunciar.

Puede apreciarse que el objeto de la pulsión, a través de su carácter instrumental, aparece como reconstituyendo en un nuevo nivel la acción específica perdida a nivel de la necesidad, designando de este modo una satisfacción propia del sujeto psicoanalítico y no del organismo biológico. Pero, también cabe recordar que Freud en modo alguno confunde esta satisfacción con la del cumplimiento del deseo, vale decir, con la identidad de percepción del proceso primario [alucinación 
desiderativa]. (RABINOVICH, 2007, p. 24. El destacado entre corchetes nos pertenece.)

Por último, tenemos lo que Freud denominó el objeto de amor, siempre definido como un objeto de amor total, y que variará entre la elección narcisista (amar al yo especular imaginario) y la elección anaclítica (elección que dependerá de la identificación con la madre o el padre, en tanto Otro mujer u hombre primordial). Como podemos notar, la definición del objeto freudiano como objeto de amor dependerá de la relación del sujeto con el falo como tercero que permite la triangulación edípica. Pero el objeto de amor se diferencia del objeto sexual, y por eso podemos afirmar que la elección homo-heterosexual depende de una cuestión de identificación anterior que va más allá de la elección del objeto sexual. Freud señala en Introducción al narcisismo (1984) que el ser humano tiene inicialmente dos objetos sexuales originarios: él mismo y la mujer que lo crió.

El primero de ellos funda la elección narcisista, el segundo la elección anaclítica. El carácter central que Freud le adjudica a la elección narcisista es su meta pasiva -ser amado- y el hecho de que todo gira en torno a los rasgos del sujeto mismo. En el caso de la elección anaclítica, vale decir de la mujer que lo crió, a la que Freud le agrega el padre protector, existe una identificación activa con alguna de estas dos figuras. Aquí el amor en su surgimiento se apoya sobre la necesidad, es decir, que Freud retoma respecto al amor la noción de apuntalamiento sobre la necesidad, al menos en lo tocante a la elección más madura, y señala también su meta activa. (RABINOVICH, 2007, p. 25)

El narcisismo es una forma de elección del objeto de amor que consiste en una actitud de adoración pasiva del sujeto hacia su propio yo. La forma anaclítica de elección de objeto, por el contrario, requiere de un trabajo activo de identificación con alguno de los 
progenitores. En el caso de la psicología de las masas, la elección amorosa de los individuos se encarna en la figura del líder, y es eso mismo lo que permite la configuración de la masa. Es decir, se da una particular superposición o solidaridad entre la identificación primaria y el líder como el modelo a seguir que el yo persigue, demarcado siempre por el Ideal del yo. Al mismo tiempo, como señala Freud para el caso de cualquier estado de enamoramiento, la coincidencia en la elección del objeto amoroso de los individuos que conforman la masa en la figura del líder supone una transferencia de la libido narcisista hacia el objeto amado, que es lo que resulta en ese estado de idealización que "falsea el juicio", típico de todo enamorado. Esta puesta en común es lo que permite la identificación posterior entre los pares o adeptos al líder de la masa. Es decir, lo que demuestra el texto de Freud es que la identificación entre los miembros que pueden formar una masa sólo es posible porque ha habido otro proceso anímico anterior, llamado elección del objeto de amor, en el que coincidieron. Los sujetos que pueden formar una masa no lo logran por identificarse con el líder y sí con sus pares, por haber puesto cada uno en el lugar del Ideal del yo al mismo objeto de amor. Lo que no deja de validar el otro hecho, de que la identificación primaria de la que resulta la elección del objeto amoroso de los sujetos sea un elemento igualmente determinante. Hacia el líder hay un estado de idealización típico del enamoramiento, versión tierna del amor donde la pulsión se encuentra inhibida en su meta. La autora señala también que la diferenciación entre "identificación" y "elección de objeto" la encontramos en Lacan articulada predominantemente con el falo y el complejo de castración (más que en su articulación con el Ideal, como puede verse en el caso del desarrollo conceptual de Freud). Este concepto de objeto de amor, ¿cómo lo desarrolla Lacan?

Según Diana Rabinovich, Lacan irá a desarrollar una nueva formulación del amor a partir de la reubicación del concepto freudiano de frustración en la dimensión simbólica (no ya en el plano imaginario). Aquí nuevamente se expresa el alcance que el desamparo y la prematuridad que Freud había identificado en el infante 
humano adquiere para la definición del objeto de amor. Pues la noción de "frustración de amor" desembocará en el concepto de "demanda". Recordemos una vez más este punto que introducimos acerca de la importancia del Otro simbólico para la subsistencia del infante, y la función simbólica que ejerce ya el grito o llanto del niño pequeño en la medida que la respuesta o no del Otro lo transforma en un "llamado", forma de vínculo anterior al que establece la demanda pero ya determinado por lo simbólico.

La posibilidad misma que tiene ese Otro de responder o no al llamado lo vincula con el par ausencia-presencia. Precisamente, es la presencia-ausencia del Otro simbólico lo que constituye al agente de la frustración en cuanto tal. El problema no es pues la presencia-ausencia del objeto real sino la presencia-ausencia de este Otro simbólico. Una vez que la necesidad atravesó el lugar del código surge transformada en demanda. (RABINOVICH, 2007, p. 123.)

La pérdida de naturalidad con el objeto de satisfacción que el psicoanálisis verifica en los seres hablantes nos introduce en el dilema de, por un lado, tener que formular demandas -que son siempre demandas de amor $-\mathrm{y}$, por el otro, en la necesidad de la frustración de la misma para poder abrir la vía del deseo. La demanda se vincula siempre a la presencia o la ausencia del Otro como el agente que detiene el poder para satisfacer. Por eso los objetos adquiridos son muestras del don, de la buena o mala voluntad, de ese Otro. Es decir, la demanda no apunta a la satisfacción de la necesidad sino a la obtención de ciertas pruebas o signos del amor del Otro.

La demanda es pues demanda no de la satisfacción de la necesidad, sino de la presencia o ausencia del Otro como el agente. Y como el Otro detenta el privilegio de poder responder o no a la demanda, ese privilegio lo dota de un poder que es el fundamento de su omnipotencia [...] El poder real que le otorga a la madre el carácter de omnipotencia 
brinda a su respuesta un valor: los objetos de satisfacción se vuelven signos de la buena o mala voluntad de ese Otro, poder en lo real, signos en última instancia de su amor. Pero, cuidado, se trata de signos de amor del Otro, no de objetos de amor, el verdadero objeto de amor es ese Otro primordial. Esos signos del amor del Otro, que transforman al objeto u objetos reales de la necesidad en objetos indiferentes desde el ángulo de la necesidad misma, son aquello que Lacan denomina con toda precisión dones. (RABINOVICH, 2007, p. 124.)

Desde esta perspectiva del objeto de amor, el pecho materno, para continuar con el ejemplo que dimos, pasa de ser el lugar de cierto goce (objeto de la pulsión parcial) a tomar el valor de ser una prueba del amor del Otro hacia el niño pequeño. Con esta formulación de los dones como objetos simbólicos que tienen valor abstracto - en sentido económico - por ser signos del amor del Otro, podemos completar la explicación que dimos al comienzo de esta sección, acerca de la alienación estructural en el orden simbólico, constitutiva de la condición subjetiva de los seres hablantes, y ver hasta qué punto no solamente la valorización del mundo sino el mismo acceso a la "realidad objetiva" depende de este enajenamiento en el significante y de la frustración de amor que genera el movimiento aleatorio y la respuesta a capricho del Otro primordial. Sucede que la imposibilidad del Otro de responder a este nivel de lo incondicional que la demanda del sujeto le plantea deja a ese Otro en vilo, dividido por su deseo, lo cual al mismo tiempo representa una herida en su omnipotencia. Pero debemos entender que la herida narcisista afecta tanto al Otro como al propio sujeto. La relación del sujeto con un objeto atravesado por el significante instala la falla-en-ser del objeto de amor (el Otro) pero también del sujeto. Al mismo tiempo, esta falla en ser es lo que les permite a ambos el desplazamiento. La frustración de la demanda de amor, de responder a título de garante de la totalización y completitud del ser del sujeto, deja al Otro en vilo, descubierto en su misteriosa dimensión de ser deseante. De este modo, una vez frustrada la demanda de amor incondicional, se 
vislumbra a ese Otro en su barradura estructural, en su ser deseante. Y cayendo como un resto no articulable en la demanda, adviene la dimensión del deseo también para el propio sujeto. El nombre que Lacan le da a ese resto no articulable en la demanda es el de objeto fálico. Es decir, el falo es el objeto regulador del deseo, del desear, el propulsor del movimiento de búsqueda al nivel significante de la recuperación de algo de ese goce absoluto por estructura perdido. El objeto fálico se presenta entonces como equivalente a la huella mnésica freudiana, como el que demarca las coordenadas de placer con las que el sujeto se orienta hacia la realización del deseo. Pero, para ser más precisos, el objeto fálico regula el deseo pero no es el objeto del deseo, en el sentido de un destino, meta, o alguna materialidad concreta a poseer. Este desprendimiento del objeto fálico que surge de la frustración de la demanda de amor viene aparejado de ese dilema edípico con el que el sujeto se topa en un determinado momento en su crecimiento. Este dilema inconsciente surge entre la identificación del ser del sujeto con el objeto imaginario faltante en la madre, ese que podría colmar su falla en ser (falo materno imaginario); o la identificación con aquél que supuestamente lo tiene o bien con aquél que supuestamente no lo tiene. La castración simbólica es la renuncia a la identificación con ese objeto fálico materno imaginario. Lo cual supone la instalación de una pendencia ontológica abierta por el sistema significante. Como indica la psicoanalista, siguiendo a Lacan, la castración es la instauración de una "deuda simbólica" (RABINOVICH, 2007, p. 133) que el sujeto paga con el falo imaginario, con la renuncia al goce ilimitado que le propiciaría la identificación de su propio ser con ese objeto fálico materno faltante. Y es precisamente esta pendencia, esta falta que se instala en el lugar de la renuncia, la que permite el advenimiento de la dimensión simbólica del deseo y su desplazamiento.

Cada vez que el sujeto cree atraparlo [al objeto de deseo] vuelve a huirle, surgiendo entonces como deseo de Otra Cosa. El deseo como deseo de Otra Cosa es uno de los nombres de la 
identidad entre metonimia y deseo. Esa Otra Cosa indica que el objeto al entrar en el círculo significante pierde, al perder su naturalidad, su valor natural, ese valor que le brindaría su especificidad en tanto que objeto de la necesidad [...] Esta dimensión del deseo de Otra Cosa es otro rostro de ese padecer -que habría que escribir padeser- propio de los seres hablantes, que es el aburrimiento, quizá por excelencia uno de los afectos más humanos. El aburrimiento nos recuerda que junto con la especificidad del objeto también se volatilizó toda posibilidad de lograr una satisfacción esencial. A ello se debe que el deseo presente esa apariencia engañosa de infinitud, y que lo mismo, lo igual, lo suman en el estasis del tedio. Su satisfacción pasará de la esencia necesaria a la contingencia del encuentro... (RABINOVICH, 2007, p.147.)

La castración simbólica coloca al sujeto en una posición de tener que intentar colmar esa falla en ser, subsanar ese afecto del aburrimiento, intentando ubicarse en ese lugar de objeto de deseo del Otro.

Ahora bien, intentemos finalmente poner este último contrapunto entre la demanda de amor incondicional y el objeto de deseo en sintonía con las demandas sociales populares que están en la base de la constitución hegemónica del pueblo en el populismo. Para ello es importante no precipitarse y establecer una pronta analogía entre la forma de Laclau y Lacan de entender a la demanda y el tipo de "mal" que la aqueja, porque de otro modo podríamos llegar a conclusiones equivocadas. Por ejemplo, no debemos entender que por detrás del concepto de demanda social hay siempre y necesariamente una demanda de amor incondicional. Es conveniente, por eso, tomar en cuenta que la relación entre los desarrollos conceptuales del psicoanálisis y la teoría política son como piezas de un puzle incompleto. Es decir, no esperar que de sus puentes y articulaciones podamos tener como resultado un todo coherente y armonioso. No obstante lo cual, y dado que además toda teoría política supone siempre una antropología o una perspectiva de la 
condición humana, creemos que vale la pena seguir intentando una aproximación analítica entre ambos campos del saber.

La analogía establecida por Laclau, entre la irrupción del líder populista mediante una investidura pulsional de su nombre y el surgimiento del objeto $a$, nos conduce a pensar al líder como un resto, un exceso que cae o que resulta de la articulación entre las distintas demandas sociales insatisfechas pero que, a la vez, no puede ser reducido a ninguna de ellas ni tampoco quedar articulado en el nivel de la equivalencia inicial. Pero esto ya lo dice Laclau en sus propios textos. Lo que nosotros queremos añadir, siguiendo los desarrollos teóricos del psicoanálisis (ALEMÁN, 2009, 2012, 2014), es que esa potestad para poder sustraerse a la articulación inicial puede darse al menos de dos modos radicalmente distintos. Por un lado, el líder puede asumir la representación del Otro de la demanda de amor incondicional, el Otro de la garantía, lo que nos lleva a pensar en la identidad política popular en términos de la masa comandada por un prefigurado ideal de completitud y perfección. Por el otro, el líder puede cobrar su valor por asumir el lugar de la representación de una causa política, de un deseo colectivo forjado más allá de las actuales demandas que la sociedad se plantea. Para esto último es necesario que sea un líder castrado, un ser deseante que haya renunciado a la omnipotente de querer responder a título de garante frente a las demandas de la sociedad, y ofrecer en su lugar el asumir la responsabilidad por su propia iniciativa y acción política. En otras palabras, para poder decir que el líder populista representa a una determinada hegemonía del mismo modo que un objeto a que cae inarticulado en la demanda, es necesario que responda con servicio y vocación a la construcción política del "menú", a la apertura de oportunidades y combinaciones entre diferentes acciones y políticas públicas reales que puedan materializar no un ideal político acabado sino una causa política abierta. Sin querer saturar de antemano la elección del propio pueblo del cual ha surgido. Retomando el ejemplo del restaurante chino que cita Lacan en el Seminario 11. Los cuatro conceptos fundamentales del psicoanálisis (2012), en la clase XX titulada "En ti más que tú", 
podríamos decir que al líder de una causa política le compete el rol del mozo que ofrece el menú pero que se niega a responder ante la pregunta que el pueblo indefectiblemente habrá de formularle: “QQué deseo yo de todo esto?, a usted le toca saberlo" (LACAN, 2012, p. 277). Es decir, cuando pensamos en el líder populista como aquél que habla desde el lugar enunciativo de una causa política estamos pensando en alguien que tiene la capacidad para alojar la demanda de saber absoluto pero al mismo tiempo de poder frustrarla, es decir, devolverla con la marcación de algún grado de incerteza. No es cuestión entonces ni de querer negar esa demanda popular pero tampoco de tener que prescindir de ella, sino de darle un lugar pero para devolverla con la marca del propio rasgo, de la propia respuesta que, como tal, será siempre parcial y contingente.

\section{Palabras finales}

Pensar el populismo en perspectiva con lo que el psicoanálisis desarrolla acerca de la tríada necesidad-demanda-deseo requiere entender la diferencia entre lo que significa la formulación de la demanda (necesaria al inicio del proceso de subjetivación) y la frustración de la misma (igualmente necesaria para la apertura a la dimensión del deseo). En este sentido, lo decisivo es entender que no habrá nunca frustración de demanda sin formulación de demanda, y que la sensación de frustración proviene de la experiencia de la falta de plenitud. Pensando en las demandas sociales que están en la base del populismo, la falta de plenitud no debe entenderse necesariamente en el sentido de que la satisfacción de las mismas nunca podrá ser alcanzada (cosas como el "pleno empleo", por ejemplo). Pues este modo de pensar la incompletitud todavía responde a un deseo de omnipotencia idealizada infantiloide. Hay también otro modo de tramitar la castración que supone la asunción de una causa política, que es mostrando y asumiendo colectivamente el costo que habrá que pagar por la realización de las propias metas. Por ejemplo, tomando consciencia de las decisiones políticas en cuanto a los pesos específicos y diferenciales que adquieren determinadas reparticiones gubernamentales o asuntos de interés público en las 
partidas presupuestarios de los Estados. Es decir, mostrando cómo algo finito - por ejemplo, los impuestos recaudados - se reparte y distribuye al interior de una sociedad atravesada por determinadas disputas políticas. En este terreno precario es donde juega su suerte la hegemonía populista del modo como la piensa Laclau. El líder populista que ejerce el liderazgo político aquí no es más que el líder de una Causa política, es decir, un Otro castrado. De otro lado, una conjugación perversa entre demandas sociales insatisfechas y delirios de grandeza puede conducir a la emergencia de otra posición, absolutamente carente de no-saber, de un liderazgo que encarne el anhelado Ideal de omnipotencia, al estilo de los neofascismos que infelizmente estamos viendo surgir en el marco de un grave declino de las democracias contemporáneas. En parte podrían ser llamados "populismos de derecha", pero en todo caso lo que intentan capturar es una verdad y una seguridad que provienen no de la forma política de la hegemonía sino de la fe en el credo religioso, en el fanatismo irreflexivo, la omnipotencia infantil y un totalitarismo pulsional de tipo pre-simbólico.

\section{Referencias bibliográficas}

ALEMÁN, J. Para una izquierda lacaniana... Intervenciones y textos. Buenos Aires: Grama Ediciones, 2009.

Soledad: común. Políticas en Lacan. Buenos Aires: Capital Intelectual, 2012.

. En la frontera. Sujeto y capitalismo: el malestar en el presente neoliberal. Buenos Aires: Gedisa Editorial, 2014. LACAN, J. El seminario de Jacaques Lacan: libro 11: Los cuatro conceptos fundamentales del psicoanálisis. Buenos Aires: Paidós, 2012.

LACLAU, E. Política e ideologia na teoria marxista. Rio de Janeiro: Paz e Terra. 1979.

; MOUFFE, C. Hegemonía y estrategia socialista.

Buenos Aires : Fondo de Cultura Económica, 2004.

La razón populista. Buenos Aires: Fondo de Cultura

Económica, 2005. 
RABINOVICH, D. S. El concepto de objeto en la teoría psicoanalítica: sus incidencias en la dirección de la cura. Buenos Aires: Manantial, 2007.

. Teóricos de psicoanálisis Escuela Francesa. Teórico n. 2, 1986. Facultad de Psicología de la Universidad de Buenos Aires. Cátedra Rabinovich, Psicoanálisis: escuela francesa. Disponível em: <http://23118.psi.uba.ar/academica/carrerasdegrado/ psicologia/informacion_adicional/electivas/francesa $1 /$ material/ Teorico\%202\%20-\%201986.pdf> . Acceso en 20 de out., 2020.

\section{Resumen:}

El objetivo general del trabajo es desarrollar un análisis que nos permita localizar y ponderar la influencia del psicoanálisis en la teoría política de Laclau, en particular para pensar la dimensión del afecto presente en su conceptualización del populismo. El populismo es para este autor una forma de estructuración de la identidad política, no solo de los agentes sociales como también de la sociedad que resulta instituida como consecuencia de la irrupción de lo político. En este sentido, el lugar enunciativo del líder populista es el que permite la totalización hegemónica de la misma, aquello que establece el horizonte de inteligibilidad política posible para una determinada coyuntura histórica específica. Laclau (2005) construye una analogía entre esta función de cerramiento, parcial y contingente, de la hegemonía populista y la función del objeto a lacaniano. Nos proponemos aquí, entonces, comenzar a explorar esta analogía.

Palabras clave: populismo, afecto, demanda, deseo, objeto a.

\section{Resumo:}

O objetivo geral do trabalho é desenvolver uma análise que permita localizar e ponderar a influência da psicanálise na teoria política de Laclau, em particular para pensar a dimensão do afeto presente na sua conceitualização do populismo. O populismo é para este autor uma forma de estruturação da identidade política, não somente dos agentes sociais como da sociedade que fica instituída a consequência da irrupção de o político. Neste sentido, o lugar enunciativo do líder populista é o que permite a totalização hegemônica da mesma, o que estabelece o horizonte de inteligibilidade política possível para uma determinada 
conjuntura histórica específica. Laclau (2005) constrói uma analogia entre esta função de fechamento parcial e contingente da hegemonia populista e a função do objeto a lacaniano. Propomo-nos aqui, então, começar a explorar esta analogia.

Palavras-chave: populismo, afeto, demanda, desejo, objeto a.

\section{Abstract:}

The general purpose of this article is to evaluate the influence of psychoanalysis on Ernesto Laclau's political theory, particularly to analyse the 'affective' dimension on his conceptualization of populism. According to Laclau, populism is a means for structuring political identity with respect not only to individuals but also to society. It arises as a consequence of the emergence of the political. In this sense, the place of enunciation of the populist leader is what makes the hegemonic totalization of society possible while defining the horizon of political intelligibility in a specific historical context. In On Populist Reason (2005), Laclau establishes an analogy between this partial and contingent elaboration of populist hegemony and the function of the Lacanian 'object $a$ '. In this article, we analyse this analogy.

Key words: populism, affective dimension, demand, desire, object $a$.

Recebido em 31 de agosto de 2019

Aprovado em 09 de junho de 2020 